\title{
Wege zu einem „,besseren Europa“: Plädoyer für eine Aufgabenkritik der Europäischen Union
}

\author{
von Joachim Jens Hesse und Thomas Fehrmann
}

\begin{abstract}
Die Europäische Union (EU) unterliegt seit annähernd vier Jahren einer wirtschaftlichen, politischen und rechtlichen Krisendiskussion. Oberflächliche und nicht selten inszenierte Konflikte zwischen allzu gläubigen Europabefürwortern und scharfen Euroskeptikern drohen dabei den Blick auf die konkrete Aufgabenwahrnehmung und damit die Leistungsfähigkeit der Union zu verstellen. Anknüpfend an einer Reihe weniger normativer als vielmehr funktionaler Defizite der Union entwickelt der Beitrag ein Modell, das den Europäisierungsprozess „vom Kopf auf die Füße“ zu stellen sucht. Dem dient die Erarbeitung eines empirisch-analytischen Kriterienkatalogs, der die diskussionswürdige Performanz der europäischen Politik erklärt und in die Forderung nach einer konsequenten Aufgabenanpassung (und deren Umsetzung) mündet. Den ubiquitären Rufen nach „mehr" oder „weniger" Europa wird ein empiriegesättigtes und handlungsorientiertes Plädoyer für ein funktionaleres und durchaus „, besseres Europa“ gegenüber gestellt.
\end{abstract}

For nearly four years, the European Union (EU) has been in crisis mode. Superficial tensions between all-too credulous pro-Europeans and harsh Euroskeptics tend to hide the urgent necessity of a debate on the performance and future tasks of the Union. This contribution outlines a procedural model to help structure such a debate. Based on specific examples, a catalogue of criteria for European tasks is developed in order to discuss possible approaches at reform as well as potential paths towards the implementation of such endeavours. In response to the ubiquitous calls for "more" or "less" Europe, this ought to be understood as a plea for a more functional "better Europe".

\section{Die EU zwischen Wollen und Können}

Die ausufernde Debatte um die Handlungs- und Zukunftsfähigkeit der Europäischen Union droht an ihrer Beliebigkeit zu scheitern. So kommt es zwar zu ubiquitären Klagen über einen Stillstand oder gar Rückschritt des europäischen Einigungsprozesses, wird auf diverse institutionelle und verfahrensbezogene 
Defizite verwiesen, die die Union einer „tödlichen Gefahr“ aussetzen ${ }^{1}$ - doch erscheint die Diskussion seltsam zirkulär. Ob institutionelles Versagen, organisationspolitisches Nicht-Handeln oder endgültiger Beleg für den Triumph kapitalistischer Überschussenergien über die Politik, die Schuldigen sind schnell ausgemacht: die zögerlichen Mitgliedstaaten, der unsolidarische Norden des Kontinents, verantwortungslose Südeuropäer, selbstreferentiell agierende Einrichtungen oder auch der entfesselte Bankensektor, sie alle weisen jeweils auf den anderen und dokumentieren eine nur sehr eingeschränkte Kooperations- oder gar Veränderungsbereitschaft.

Damit wächst die Polarisierung und kommt es zu Verteilungskämpfen bislang ungeahnten Ausmaßes (und diskussionswürdigen Stils). Zudem wird die Distanz zu den „Völkern Europas“, auf die man sich in den Europäischen Verträgen beruft, immer deutlicher. Sich darauf richtende Ursachenanalysen bleiben aus oder werden dem erreichten Komplexitätsgrad des Europäisierungsprozesses kaum mehr gerecht, die Ausdifferenzierung einzelner politisch-administrativer, ökonomischer oder soziokultureller Verwerfungen verstärken diesen Prozess. In der Folge findet sich verbreitet Ratlosigkeit, zudem eine Rückkehr zu bereits mehrfach gescheiterten appellativen Politiken. Danach sei die Krise inzwischen „gemeistert“, ginge es lediglich darum „zu lernen“ und sich künftig veränderter Verhaltensmuster zu befleißigen. Je nach Standort scheiden sich dabei einmal mehr die begeisterten, um nicht zu sagen geradezu „gläubigen“ Europäer von denjenigen, die dem strittigen „Wollen“ der Europäischen Union auch ein „Können“ entgegenzusetzen suchen.

Trotz unbestreitbaren Bemühens politischer Akteure um problemangemessene Reaktionen erfährt das Publikum im Ergebnis ein eigenartiges déjà-vu. Entsprechend mäandert die EU zwischen Nord und Süd, Ost und West, Zahlmeistern und freeridern, Claqueuren und Querulanten oder auch „Berufseuropäern“ und „Euro-Skeptikern“, während nüchterne Beobachter auf Diskussionswürdiges verweisen, ohne die Leistungen im Rahmen des historisch angezeigten und umfassend gewollten europäischen Einigungsprozesses kleinzureden. Auch sie freilich dokumentieren fragwürdige Professionalisierungsprozesse und demokratiepolitische Schwächen, samt der sich damit verbindenden Legitimationsdefizite. Meist wird dabei übersehen, dass die Krise durchaus von neuer Qualität ist, sie substantiellere Reaktionen als bisher erfordert.

1 „Die Europäische Union in der Krise: Auswege und Handlungsoptionen“ - Martin Schulz im Gespräch mit Joachim Jens Hesse, in: ZSE, 11/1 (2013), 143-152, hier 143. 
Schon das abschwächende Argument, die EU befinde sich seit ihrer Begründung in einer Art „Dauerkrise“, kann kaum überzeugen. So wurden zahlreiche der bisher durchlebten Krisen ja durchaus bewusst herbeigeführt, sei es aufgrund der strategischen Zuspitzung durch mindestens einen Mitgliedstaat oder aber im Zuge der Verfolgung gruppen- und in Einzelfällen personalbedingter spezifischer Interessen. Dieses gleichsam endogene Entwicklungsprinzip hat sich verstetigt und gleichsam strukturell verfestigt: die EU als politisch forciertes und (in wesentlichen Teilen) auch legitimiertes, aber kontinuierlich kompromissbehaftetes und nur bedingt krisenresistentes Konstrukt.

Seit dem Jahr 2009 finden sich dagegen erstmals ernsthafte unintended consequences und ein institutionelles Versagen in bislang nicht beobachtetem Ausmaß. Die Entwicklung der vergangenen vier Jahre stellt eine qualitativ neue Herausforderung dar, zumal ihre Ausprägung bei entscheidenden Akteuren zu einer Neubewertung der bisher allseitig erkennbaren „Kooperationsrendite“ geführt hat. Aus dieser Sicht wird es Zeit, die gegenwärtige Krise als das zu verstehen, was sie tatsächlich darstellt: einen exogen erzwungenen Austausch über Zielkonflikte, die im Tagesgeschäft bislang übertüncht wurden. Parallel dazu und damit meist unverbunden findet sich eine Diskussion, die jenseits wohlfeiler Absichtserklärungen wesentlich nachhaltiger als bislang versucht, eine europaweite Öffentlichkeit zu fördern, sei es über die Herausbildung grenzund ebenenübergreifender Diskurse (zur Verschränkung der nationalen Öffentlichkeiten), sei es über den aktiven Einbezug der Akteure europäischer Einrichtungen. Letzteres macht wieder Hoffnung auf eine auch analytischere Sichtweise, die sich mit den angesprochenen Entwicklungsprozessen verbindende (themen- und akteursspezifische) Segmentierung des politischen Verhaltens wie der Öffentlichkeit in das Zentrum zukunftsorientierter Untersuchungen zu rücken, die Krise deshalb auch als Ausgangspunkt sich selbst verstärkender Kooperationen zu sehen.

Diese unterschiedlichen Argumentationsstränge zusammenfassend wird deutlich, dass zu den erkennbaren normativen Auseinandersetzungen eine verstärkt funktionale Diskussion treten sollte, die der Frage nach dem „Sollen“ der EU jene nach ihrem „Können “ gegenüberstellt. So übersieht die eher normativ ausgerichtete Debatte, dass die Forderung nach „mehr Europa“ eher die Herausbildung antieuropäischer oder zumindest euroskeptischer Haltungen befördert, die in vielen traditionell europafreundlichen Mitgliedstaaten derzeit deutlich anwachsen und für die Wahlen zum Europäischen Parlament düstere Prognosen zulassen - während die eher funktionale Diskussion auf die Leistungen und Arbeitsweisen 
der europäischen Einrichtungen abstellt und sie einer Art von Wirkungsforschung zuführt. Im Ergebnis scheint es an der Zeit, die so legitimen wie notwendigen Diskussionen um die normative Basis der Union sich nicht verselbständigen zu lassen, sie vielmehr diesen funktionalen Realitäten zu konfrontieren. Die Beantwortung der Frage „Was kann die EU?“ wird mithin zwischen überbordendem Integrationseifer und der Gefahr, das Kind mit dem Bade auszuschütten, vermitteln müssen. Wendet man die europapolitische Auseinandersetzung in diese Richtung, stellen sich als Handlungsalternativen entweder die Anpassung der wahrgenommenen bzw. wahrzunehmenden Aufgaben oder aber die Veränderung des institutionellen Rahmens wie der einzusetzenden Instrumente - was wiederum die Notwendigkeit beinhaltet, Europa als gebietskörperschaftliche Ebene mit eigenem Aufgabenzuschnitt zu begreifen.

Da die Überwindung nationalstaatlicher Egoismen, geschweige denn die des Nationalstaats per se, auf absehbare Zeit ebenso wenig zu erwarten ist wie der Übergang zu einem europäischen Bundesstaat, verbleibt für den Moment allein die Möglichkeit einer den Namen verdienenden Aufgaben- und Leistungsüberprüfung (vulgo: Aufgabenkritik), bevor man wieder Vertragsänderungen das Wort redet oder gar die erneute Einsetzung eines Konvents favorisiert, um der erkennbaren Probleme Herr zu werden.

\section{II. Überforderter Nationalstaat - überfordertes Europa? Zur funkti- onalen Grundierung einer normativen Diskussion}

Nicht ohne Grund verweist die analytisch anspruchsvollere Diskussion zum laufenden Europäisierungsprozess immer wieder auf die mit seiner Fortentwicklung verbundenen economies of scale und economies of scope. Gemeint sind jene Größen- und Verbundvorteile von Integrationsprozessen, die so unabweisbar wie unverzichtbar sein sollten, im Rahmen der Europäischen Union aber aufgrund eher ganzheitlicher Sichtweisen und der Betonung des sui generis-Charakters des Europäisierungsprozesses eher unterausgeschöpft erscheinen. Dies spiegelt sich deutlich im Prozess der Politikformulierung und dessen Vollzug. Arbeitsfähige, horizontal und vertikal aufdifferenzierte Organisationsstrukturen werden eher neu konstruiert, während bewährte und „gelebte“, also nicht nur kodifizierte, sondern auch operativ greifende Verhaltensmuster subsidiärer Einrichtungen als nachrangig eingeschätzt werden. Nicht von ungefähr konzentrieren sich die handelnden Akteure eher auf Kommunikation und (häufig nur sehr „weiche“) Kooperation statt auch koordinativen und damit im Ergebnis letztlich arbeitsteiligen Verfahren das Wort zu reden und sie politisch-administrativ umzusetzen. Damit 
entzieht man sich den „Mühen der Ebene“, greift erneut zu großformatigen Handlungsempfehlungen anstelle notwendigerweise ausdifferenzierter Aufgabenzuordnung - mit der Folge einer unzureichenden Rückbindung an die ,Völker Europas“ und der über diese gewährleisteten demokratiepolitischen Minima. Im Ergebnis stehen dem normativen Überschuss der Diskussion beträchtliche funktionale Defizite gegenüber.

In diesem Kontext scheint es angezeigt, einer Ende der 1990er Jahre für die Nationalstaaten diagnostizierten „Überforderung des Staates“2 auch die Frage nach einem potentiell überforderten Europa gegenüberzustellen. Die Verbindungslinien sind deutlich und bieten sich für eine gewisse Verallgemeinerung durchaus an. Im Zentrum steht das Dilemma mangelnder Aufgabenangemessenheit der politischen Willensbildung. Solange die Mitgliedstaaten etwa Aufgaben auf die Union übertragen, die Instrumente zu deren Erfüllung aber fast immer unter Vorbehalt stellen, muss die EU im Vollzug vor Schwierigkeiten stehen. Dies korrespondiert dem Verhältnis von Zentral- und Gliedstaaten in den föderativen politischen Systemen Europas. Hinzu treten Grenzen der europäischen Integration, die man als finalité implicite bezeichnen könnte. Sie verweisen auf defizitäre Größen- und Verbundeffekte (dis-economies of scale and scope), die etwa in der bislang fast vollständigen Nicht-Berücksichtigung des Subsidiaritätsprinzips zum Ausdruck kommen - und im Übrigen auch in den Vereinigten Staaten unter der Chiffre dysfunctional democracy diskutiert werden. Dass dabei die Grenzen nationaler Verfassungssysteme zu beachten sind, ist ausreichend diskutiert, nicht zuletzt am Beispiel Deutschlands und Großbritanniens. Ein Ausweg böte sich letztlich nur, wenn man das Können wie auch das NichtKönnen der EU empirisch-analytisch konsequent zu erfassen suchte, etwa durch den Ausweis angemessener und durchaus erfolgreicher europäischer Politiken, aber eben auch unter Berücksichtigung jener inflationären „Prozesse“, die man seitens der Kommission über die Jahrzehnte zu verfolgen sucht. Dies zu systematisieren, den gelungenen auch die unangemessenen, misslungenen und erfolglosen europäischen Ansätze gegenüberzustellen, wäre einige Anstrengungen wert. Eine solche systematische Erörterung hätte zudem den Vorteil, dass man die Ebene der Einzelfälle verlassen und sich systematisch nicht nur der Aufgabenangemessenheit, sondern auch der Aufgabentragfähigkeit der Europäischen Union zuwenden könnte. Der analytische Zugang wird so vom Sollen über das Können bis hin zur tatsächlichen Ausübbarkeit geführt - jeweils unter Berücksichtigung 
der rechtlichen und politischen Rahmenbedingungen für das Handeln der Europäischen Union.

Folgt man dem, könnte sich die Krise der vergangenen Jahre als ein „Weckruf“ erweisen, da sie die Chance bietet, das Bewährte, Machbare und Akzeptierte zu stabilisieren und zu konsolidieren. Die Anerkenntnis früherer Fehler und institutionellen Versagens (nationalstaatlich wie europäisch) verbände sich mit einer aufgabenspezifischen Bestandsüberprüfung (mit den Zielen: Beibehaltung, Ausbau oder Rückbau) sowie möglichen Tragfähigkeitsanalysen (europäisch wie mitgliedstaatlich), die wiederum den Ausweis organisationspolitischer Konsequenzen erlaubten. Eine solche Analyse führte im Übrigen über den Bereich der EU hinaus und hätte jenseits von Zentralstaat, gliedstaatlichen Einrichtungen und kommunaler Ebene auch den Einbezug von Internationalen Organisationen zum Gegenstand. Ressourcenbezogene Konsequenzen schlössen sich an, von der Steuerverteilung über einen etwaigen europäischen Finanzausgleich bis hin zur künftigen Rolle der Europäischen Zentralbank und des Internationalen Währungsfonds. Im Ergebnis stünde nicht nur eine verbesserte Form der Aufgabenwahrnehmung für die Bürgerschaft, den Unternehmensbereich und letztlich auch den Staat selbst, sondern auch ein Abbau destruktiver Vorurteile gegenüber einer regelmäßigen Überprüfung der jeweiligen Aufgabenbestände.

\section{Aufgabentragfähigkeit als entscheidende Kategorie}

Eine vor allem unter funktionalen Gesichtspunkten zunehmend staatsanalog wirkende Europäische Union sollte mithin den gleichen Überprüfungsprozessen unterliegen, die sich in der Vergangenheit für national- und gliedstaatliche Regierungs- und Verwaltungssysteme bewährt haben. Allerdings ist bislang ein deutliches diskursives Ungleichgewicht zwischen den daran zu beteiligenden Disziplinen zu beobachten: Die staats- und europarechtliche Diskussion samt ihrer normativen Prämissen wird im Rahmen der EU meist nur um einen ökonomischen Diskurs ergänzt, der sich zum einen auf die europäische Wirtschaftspolitik, zum anderen aber auch zunehmend auf die individuellen Vorteils- und Nachteilskonstellationen der (und für die) einzelnen Mitgliedstaaten richtet. Zwar ist die Herausbildung rechtlicher und politökonomischer Schwerpunkte in der öffentlichen Diskussion als ein nachvollziehbares Ergebnis des europäischen Integrationsverlaufs zu werten, doch führten sie gerade in der deutschsprachigen Öffentlichkeit zu einer erkennbaren materiellen wie disziplinären Asymmetrie. Während in einigen anderen Mitgliedstaaten, vor allem in Großbritannien, die Angemessenheit des europäischen Aufgabenumfangs zu den Kernthemen der 
(durchaus ernsthaft und sachbezogen geführten) politischen Auseinandersetzung zählt, kommt derartigen funktionalen Betrachtungen in Deutschland eine nur geringe Aufmerksamkeit zu.

Gerade angesichts der funktionalen Staatsähnlichkeit der EU bedarf diese einseitige Schwerpunktsetzung jedoch dringender Ergänzung. Während staatsrechtliche und wirtschaftspolitische Diskurse auf nationalstaatlicher Ebene stets von Überlegungen zur Notwendigkeit, Angemessenheit und Verteilung öffentlicher Aufgaben auf unterschiedliche gebietskörperschaftliche Ebenen flankiert werden, sieht sich die europäische Ebene kaum systematischer Aufgabenkritik ausgesetzt. Dabei könnte die Erfahrung föderalstaatlicher Aufgabenteilung und -verflechtung von beträchtlichem Wert sein, sucht man das europäische Regierungsund Verwaltungssystem zukunftsfähig zu gestalten; ${ }^{3}$ frühere Arbeiten haben bereits auf mögliche Parallelitäten verwiesen. ${ }^{4}$ Gerade das Mehrebenensystem der EU bedingt eine konsequente Aufgabensystematisierung und -analyse, da nur so eine Balance von Kooperation und Wettbewerb, Koordination in Planung und Vollzug, Aufwand und Ertrag oder auch Reform und Stabilität angemessen erörtert werden kann. Daher wäre zu prüfen, ob entsprechende Ansätze zur Systematisierung im staatlichen und kommunalen Bereich ${ }^{5}$ auf die Europäische Union ausgeweitet werden können, um eine vergleichbare, empirisch-analytisch anspruchsvolle und belastbare Grundlage für weitergehender Reformbemühungen zu schaffen. ${ }^{6}$

Einen ersten Schritt zu einer solchen Aufgabenüberprüfung auch auf europäischer Ebene stellt die Betrachtung der institutionellen Tragfähigkeit der Union dar. Sie ermöglicht die nachfolgend exemplarisch durchgeführte Entwicklung eines Kriterienkatalogs, auf dessen Grundlage in weiteren Schritten der Aufgabenzuschnitt auf seine Angemessenheit hin untersucht werden soll. Der Erarbeitung eines solchen Kriterienkatalogs kommt im Rahmen von Aufgabenüberprüfungen große empirisch-analytische Bedeutung $\mathrm{zu}$, da erst auf seiner Basis belastbare Erkenntnisse zu gewinnen sind. Die hier verfolgte Bewertung orientiert sich am Status quo und der grundlegenden Prämisse, dass eine aktuellere

3 Scharpf, F.W.: Optionen des Föderalismus in Deutschland und Europa, Frankfurt a.M., 1994.

4 Hesse, J.J.: Die bundesstaatliche Ordnung als Strukturprinzip und Gestaltungsaufgabe: Zur Parallelität der deutschen und der europäischen Föderalismusdiskussion, in: Büttner, T. (Hg.): Finanzverfassung und Föderalismus in Deutschland und Europa, Baden-Baden, 2000, 9-38.

5 Hesse, J.J.: Aufgabenkritik, Funktional- und Strukturreform in den Flächenländern, Baden-Baden, 2007.

6 Vgl. u.a. die Ausführungen des Parlamentspräsidenten Martin Schulz in einem sich u.a. auf diese Fragestellung bezogenen Interview, in: ZSE 11/1 (2013), 143-152, 149. 
Reform des europäischen Aufgabensystems, mithin der Kompetenzordnung, angesichts der verfassungsrechtlichen Rahmenbedingungen und des erkennbar mangelhaften politischen Rückhalts in einigen Mitgliedstaaten ohne weitere Transfers nationalstaatlicher Souveränität auf die europäische Ebene auskommen sollte. Davon ausgenommen sind lediglich punktuelle Anpassungen, die zu einer optimierten Wahrnehmung bestehender Aufgaben unausweichlich sein dürften. ${ }^{7}$ Die nachfolgend anhand ihrer organisatorischen, prozessualen und ressourcenbezogenen Dimensionen untersuchte institutionelle Tragfähigkeit bildet auch insofern die Basis für eine weiterführende Diskussion funktional sinnvoller Reformansätze.

\section{Organisatorische Tragfähigkeit}

Mit Blick auf die Organe und Einrichtungen der Union ist inzwischen ein ausbalanciertes System erkennbar, das durchaus von einer nachweisbaren Fähigkeit zum Ausgleich unterschiedlicher Interessen gekennzeichnet ist - sei es zwischen einzelnen Mitgliedstaaten, sei es bei Konflikten zwischen der europäischen und der mitgliedstaatlichen Ebene. Diese Ausgleichsfunktion wird zurecht gelobt und stellt vor allem in der Außenwirkung der EU ein nicht zu unterschätzendes Kapital dar; ein Beispiel hierfür bildet in jüngerer Zeit die moderierende Wirkung der Außenbeauftragten Ashton in den E3+3-Gesprächen zum iranischen Atomprogramm. ${ }^{8}$ Auch für Fälle eines politischen und/oder rechtlichen gridlock ist die Union, anders als die meisten überstaatlichen Organisationen, seitens des EuGH mit einer wirkungsvollen (wenn auch meist einseitig integrationsfördernd wirkenden) Schiedsinstanz ausgestattet.

$\mathrm{Zu}$ dem Interessenausgleich tritt eine erkennbare Informationsfunktion, die wohl am deutlichsten im veränderten Funktionsverständnis bilateraler diplomatischer Beziehungen unter den Mitgliedstaaten zum Ausdruck kommt: Stellten sie noch vor wenigen Jahrzehnten die primären wechselseitigen Informationskanäle dar, so erschöpft sich ihre Aufgabe heute meist in einer erweiterten Repräsentation, ergänzt um Berichterstattungen über mediale und gesellschaftliche Diskurse. Die

7 Zwar wurde das mit dem Konzept funktionaler spill-over verbundene Prinzip vor allem in früheren Jahrzehnten als eine Antriebsfeder des Integrationsprozesses angesehen (vgl. Haas, E.B.: The Uniting of Europe, 2nd ed., Stanford, 1968), doch dürfte heute mit Blick auf die allseitige Bekanntheit des Phänomens, vor allem unter den mitgliedstaatlichen Regierungen, eher von einer übermäßigen Vorsicht gegenüber dieser Art des Aufgabenzuwachses auszugehen sein.

8 Manners, I.: Normative Power Europe: A Contradiction in Terms?, in: Journal of Common Market Studies 40/2 (2002), 235-258. 
grundlegenden (politisch-administrativen) Informationsfunktionen werden dagegen auf europäischer Ebene wahrgenommen, entweder über direkten intergouvernementalen Austausch (bis hin zu den regelmäßigen Treffen der Fachminister und Regierungschefs) oder aber über die Informationsdienste der Europäischen Kommission bzw. spezialisierter Einrichtungen (EZB, Eurostat etc.). Damit wiederum verbindet sich eine besondere Eignung der Union zur Überwachung harmonisierter Mindeststandards, vor allem dann, wenn damit keine zusätzlichen personellen und technischen Voraussetzungen verbunden sind.

Schließlich hat sich über die Jahre eine gewisse Ersatzfunktion insofern herausgebildet, als nationalstaatliche Regierungen eher unbeliebte Vorhaben ,über die Bande" der EU umzusetzen oder unzureichende Strukturen im eigenen Land zu ergänzen suchen. Jüngere Beispiele sind vielfach erkennbar: Im Rahmen der Regelungen zur Dienstleistungsrichtlinie, zum europäischem Haftbefehl oder zur sog. Vorratsdatenspeicherung schafften sich die Mitgliedstaaten den Freiraum, in heimischen Auseinandersetzungen nötigenfalls auf die bindende Wirkung ,Brüsseler Vorgaben“ zu verweisen, während etwa die europäische Forschungsförderung in manchem neuen Mitgliedstaat Mittel- und Osteuropas weniger als Ergänzung des Vorhandenen als vielmehr als Ersatz eigener Politiken gilt.

Andererseits erfüllen die europäischen Organe und Einrichtungen diese Grundfunktionen zumindest derzeit nur unter Inkaufnahme deutlicher Überschussenergien und Parallelstrukturen. Wer eine ausgleichend, informierend und ggf. ersetzend wirkende Kommission will, darf sich über die in beachtlicher Regelmäßigkeit zutage tretenden institutionellen Eigeninteressen und Grenzüberschreitungen nicht wundern. Wer einen Gerichtshof vorhält, um ein einheitliches und rechtsstaatliches Regelungssystem zu garantieren, darf nicht davon ausgehen, sich keinerlei unintended consequences im Rahmen autonomer Rechtsfortbildung ausgesetzt zu sehen. Hinzu tritt eine ausgeprägte Vollzugsschwäche der europäischen Ebene, verursacht durch den prinzipiellen Umsetzungsvorbehalt der Mitgliedstaaten und den damit verbundenen Mangel an umsetzungsrelevanten Befugnissen und Instrumenten. Während Nichtumsetzung und Regelverstoß in bundesstaatlichen Systemen durch ,weiche“ und ,harte“ Formen der Steuerung etwa den Einbehalt von Finanzmitteln oder die Nutzung föderaler Vollzugsformen bis hin zum Bundeszwang - bestraft (bzw. durch deren Androhung verhindert) werden können, stehen ähnlich effektive Formen im europäischen Regierungssystem nicht zur Verfügung. Der Regelbruch ist somit im Einzelfall mit suboptimalen Kostenstrukturen verbunden, was zu den als moral hazard und adverse selection bezeichneten Verhaltensweisen führen kann - etwa im Fall 
Griechenlands. Die organisatorische Tragfähigkeit ist insofern vor allem in Teilbereichen mit ausgeprägtem und unmittelbarem Vollzugsbedarf nur sehr eingeschränkt gegeben. Dabei ist weniger von einer Unterscheidung nach Politikfeldern auszugehen, als vielmehr von einer solchen zwischen Politikformulierung und administrativer Umsetzung. Während das System der Politikformulierung auf europäischer Ebene im Wesentlichen den Anforderungen gewachsen zu sein scheint, kann von einem wirksamen und effizienten Vollzug kaum die Rede sein.

Schließlich ergibt sich eine maßgebliche Beschränkung der funktionalen Tragfähigkeit europäischer Einrichtungen aus der mangelhaften Legitimität ihres Handelns. Dies richtet sich nicht nur auf das vielfach diagnostizierte Demokratiedefizit der Union, sondern auch auf einen rapiden Rückgang ihrer (wahrgenommenen) output-Legitimität im Zuge der europäischen Wirtschafts-, Wettbewerbsfähigkeits- und Staatsschuldenkrise. Konnte bislang noch argumentiert werden, dass der erzielte Ertrag jeden Mangel an demokratischer Beteiligung der Völker Europas rechtfertigt, so wird es zunehmend schwerer, den Bürgern erweiterte Belastungen und Verbindlichkeiten (sowohl in den Nehmer- als auch in den Geberstaaten) bei gleichzeitigem Verzicht auf demokratische Mitwirkung verständlich zu machen. Insofern ist vor allem bei starken politischen Gegensätzen zwischen Mitgliedstaaten und/oder Unionsorganen sowie bei grundlegenden oder tiefgreifenden Fragen politischer Gestaltung - das Bundesverfassungsgericht suchte das in seiner Lissabon-Entscheidung erstmals zu systematisieren von einem grundsätzlichen Legitimationsdefizit auszugehen, das die Aufgabentragfähigkeit der Union in der kurzen und mittleren Frist deutlich einschränken dürfte.

\section{Prozessuale Tragfähigkeit}

Auch mit Blick auf die eingesetzten Verfahren in Politikformulierung und Vollzug ist von einer nur begrenzten Aufgabentragfähigkeit der EU auszugehen. Parallel zu den angesprochenen Ausgleichs-, Informations- und Ersatzfunktionen der europäischen Einrichtungen zeichnet sich der politisch-administrative Prozess der Union durch eine ausgeprägte Orientierung am Konsens, mithin an der Befähigung zum Kompromiss, aus. Summiert wird dies bekanntlich unter dem Begriff der Gemeinschaftsmethode, die im Gegensatz $\mathrm{zu}$ anderen Verfahrensformen (intergouvernementale Beschlussfassung, offene Koordinierung) weiterhin als Standardverfahren der Union zu kennzeichnen ist. Hierbei wird, neben den formal erforderlichen Mehrheiten, der Konsens unter den Mitgliedstaaten jeder Form von Konfrontation vorgezogen. In der Folge kommt es einerseits zu 
ausgeprägten Tausch- und Koppelgeschäften, andererseits aber auch zu einer frühzeitigen Ausklammerung hochgradig strittiger Fragestellungen, etwa um Kampfabstimmungen im Rat oder im Parlament zu vermeiden.

Einen bevorzugten Modus stellt dabei die Technisierung der Entscheidungsfindung dar, vor allem durch die Erörterung und Bearbeitung legislativer und regulativer Vorschläge auf der Arbeitsebene der Kommission, des Parlaments und der Mitgliedstaaten. Dieser Aspekt des ,europäischen Regierungssystems“ stellt sicher, dass Rat und Parlament in der überwiegenden Mehrzahl der Fälle nur mit beschluss- und konsensfähigen Vorschlägen auf der Tagesordnung konfrontiert werden. Ergänzt wird dieser funktionale Fokus durch die umfassende Einbindung privater Akteure, vor allem im Rahmen formalisierter Konsultationsverfahren. Dieses Vorgehen führt zwar unzweifelhaft zu beträchtlichen Informationsgewinnen und einer umfassenden Betrachtung des konkreten Regelungsgegenstands (ggf. auch über das auf nationalstaatlicher Ebene erreichbare Maß hinaus), doch erweist es sich zugleich als in hohem Maße ungeeignet, mit tiefgreifenden und genuin politischen Grundfragen umzugehen oder gar kurzfristige Reaktionen zu ermöglichen.

Insbesondere der letztbenannte Aspekt stellt eine deutliche Einschränkung der prozessualen Tragfähigkeit dar: Durch die geringe Reagibilität der eingespielten Verfahren bedarf es vor allem in Krisensituationen einer verstärkten intergouvernementalen ad-hoc-Entscheidung. Die wachsende Nutzung zwischenstaatlicher Routinen sowie die damit verbundene Exekutivlastigkeit europäischer Politik sind aber eher Kennzeichen internationaler Politik denn staatlichen Handelns. In der jüngeren Vergangenheit wurde diese schleichende Ergänzung der Gemeinschaftsmethode, sie begann bereits in den 1980er Jahren, stark forciert. Ob im Rahmen der Gemeinsamen Außen- und Sicherheitspolitik (GASP) seit den frühen 1990er Jahren, mit Blick auf die Stärkung des Europäischen Rats durch den Vertrag von Lissabon oder bei der Schaffung des Fiskalpakts im Rahmen der Krisenpolitik der vergangenen Jahre, die Entwicklung weist in Richtung einer von den mitgliedstaatlichen Regierungen dominierten europäischen Politik. Zwar ist ein solcher Reaktionsmodus seit geraumer Zeit auch auf nationalstaatlicher Ebene beobachtbar, doch kann er hier - anders als auf europäischer Ebene - auf flankierende und ausgleichende Mechanismen zurückgreifen. Dazu zählen etwa verbindend wirkende Parteiensysteme, die auf der Basis vorab verabschiedeter Programme für zahlreiche Situationen normative Zielvorstellungen vorrätig halten und diese ebenenübergreifend einzufordern vermögen, oder aber die im 
Vergleich zu 28-köpfigen Räten und Ausschüssen weniger stark ausgeprägte Konsens- und Kompromissfindung.

Sucht man diesen Befund mit Blick auf die prozessuale Tragfähigkeit zu summieren, wird erkennbar, dass einer Ausweitung der auf europäischer Ebene verhandelten Politikbereiche und Detailfragen stets eine gewisse Verfahrensanpassung mit Blick auf Vollzugsfragen gegenüber steht. Zwar verfügt die Kommission, wie aufgezeigt, in kaum einem Bereich über ausgeprägte Umsetzungskapazitäten, doch ist ihre Position als überwachende, aktiv mahnende und ggf. klagende Instanz im Rahmen der Gemeinschaftsmethode unstrittig. Bei intergouvernementalen Prozessen spielt die Kommission hingegen eine nur nachgeordnete Rolle, die mit der eines technischen Sekretariats vergleichbar ist. Kommt es nun zu einer nachhaltigeren Verlagerung von der Gemeinschaftsmethode hin zu verstärkter Intergouvernementalität - was zunächst wertungsfrei als gegenwärtiger Trend festgestellt werden kann -, so verliert die Europäische Union im Ergebnis weitere Vollzugskompetenz und verschiebt sich der Fokus noch stärker in Richtung einer koordinierten Politikformulierung bei einzelstaatlicher Umsetzung.

Insofern scheint die Union kaum in der Lage, in kürzerer und mittlerer Frist vollzugsintensivere Aufgaben zu übernehmen. Ihre prozessualen Stärken liegen gerade nicht in Umsetzungsfragen, sondern in der Bündelung und Koordination von nationalstaatlichen Willensbildungs- und Entscheidungsprozessen. Dadurch neigt sie zur Schaffung von Lösungen, die die Politikformulierung europäisieren, während die notwendigen Vollzugsinstrumente nationalstaatlich verortet bleiben. Tragfähigkeitsbeschränkungen ergeben sich somit vor allem in jenen Politikbereichen, die schneller Reaktionsfähigkeit, eines gewissen internen Wettbewerbs oder aber einer Teilhabe an unter nationalstaatlichem Vorbehalt stehenden Vollzugsrechten bedürfen.

\section{Ressourcenbezogene Tragfähigkeit}

Schließlich muss auch mit Blick auf die zur Verfügung stehenden bzw. zur Verfügung gestellten Ressourcen von einer eher eingeschränkten Tragfähigkeit der Union ausgegangen werden. Gerade die finanziellen und personellen Kapazitäten erweisen sich als kaum noch aufgabenangemessen, da einem stetigen Aufgabenzuwachs ein inzwischen deutlich ,gedeckelter" Haushalt gegenübersteht. Entgegen den Schreckensszenarien einer überbordenden Brüsseler Bürokratie ist freilich festzuhalten, dass die Union mit ihren etwa 45.000 Bediensteten einen 
Binnenmarkt von mehr als 500 Mio. Einwohnern - samt zahlreicher angrenzender Politikbereiche - reguliert und verwaltet. So arbeiten etwa 25.000 Personen bei der Kommission im engeren Sinne - eine Zahl, die etwa im Vergleich zur Stadtverwaltung der Landeshauptstadt München, die ca. 33.000 Bedienstete umfasst, die Rede von einer allzu üppigen Ausstattung der europäischen Ebene erkennbar relativiert. ${ }^{9}$ Die jüngste Finanzplanung für die Jahre 2014 bis 2020 verdeutlicht zudem, dass die Mitgliedstaaten angesichts der zunehmend weniger kalkulierbaren Risiken der Euro-Stabilisierung kaum mehr bereit sind, weiter in „ihre“ Regionalorganisation zu investieren. Diese Entwicklung spiegelt das Stärken-Schwächen-Profil der Unionsorgane sowie die angesprochene Verfahrensverschiebung hin zu verstärkter Intergouvernementalität wider. Da der Aufbau einer vertikal vervollständigten und vollzugstauglichen europäischen Verwaltung weder erwünscht noch praktikabel ist, konzentriert man sich in Reaktionen eher auf die angesprochenen Funktionen des Interessenausgleichs, der Informationsdiffusion und des Ersatzes für nationalstaatlich nicht oder nur schwer erreichbare politische Entscheidungen.

Jenseits materieller Ressourcen verfügt die Union aber über ein beträchtliches Vermögen zum Konflikt- und Krisenmanagement. So versehen die etablierten Verfahren und ausgleichend wirkenden Einrichtungen die EU durchaus mit dem Potential, erreichbare Kompromisslinien nicht ungenutzt brachliegen zu lassen zumal einige Beteiligte häufig Funktionen übernehmen, die im Völkerrecht als das Leisten ,guter Dienste“ bekannt sind, etwa im Rahmen der Vermittlung, der Mediation und des Ausgleichs. Grundlage der in diesem Kontext ausgeprägten Tragfähigkeit (zur Überwindung divergierender politischer Zielvorstellungen der Mitgliedstaaten) ist die sog. Kooperationsdividende, die in der Vergangenheit stets dazu beigetragen hat, dass sich die EU-Mitgliedschaft für die beteiligten Akteure auch unter Heranziehung harter materieller Maßstäbe „lohnt“. Im Gegenzug muss allerdings bezweifelt werden, ob und inwieweit die europäische Politik unter dauerhaftem Verzicht auf Kooperationsdividenden tragfähig bleiben kann. Sollten sich die im Rahmen der Krise erkennbaren Umverteilungsprozesse verstetigen (was auch ohne das worst-case-Szenario einer „Dauersubventionierung eines europäischen Mezzogiorno“10 möglich wäre), käme es vermutlich zu

9 Dies gilt allerdings weniger für die immer wieder und in Teilen zu Recht kritisierten europäischen Besoldungskategorien, die einer dringenden Überprüfung bedürften. So verständlich das Festhalten an einmal gewonnenen Besitzständen auch erscheinen mag, so fragwürdig gestalten sich die Differenzen zu den im öffentlichen Dienst der meisten Mitgliedstaaten erzielbaren Einkommen.

10 Scharpf, F.W.: Die Eurokrise. Ursachen und Folgen, in: ZSE 11/1 (2013), 324-337, 336. 
einem beschleunigten Legitimationsverlust und würden in Geber- wie Nehmerländern europakritische Parteien und deren Vertreter zunehmend den Ton angeben. Gerade weil die EU keinen unauflösbaren staatlichen Herrschaftsverbund darstellt, sind die beteiligten Staaten - anders als etwa der Norden und der Süden Italiens - nicht dauerhaft an ihre Mitgliedschaft gebunden. Insofern muss die EU im Rahmen ihres Aufgabenzuschnitts solche Politikfelder meiden, die mit größeren und einseitigen Umverteilungsprozessen verbunden sind - was die Aufgabentragfähigkeit wiederum stark eingrenzt.

\section{Grundzüge einer funktionalen Aufgabenanalyse auf europäi- scher Ebene}

Summiert man die bisherigen Erkenntnisse, so müssen europäisch wahrgenommene Aufgaben insbesondere drei Kriterien erfüllen, um unter Berücksichtigung der angesprochenen Stärken wie angesichts einer mittelfristig kaum zu erwartenden Tragfähigkeitserweiterung umsetzungsfähig zu sein und zukunftsfähig zu werden:

- primäre Ausrichtung auf Fragen der Politikformulierung, der Standardsetzung, der Koordinierung und der Harmonisierung;

- Verzicht auf vollzugsintensive Aufgaben sowie auf Entscheidungskompetenzen, sofern diese konkreter Kontrollbefugnisse bedürfen, die auf nationalstaatlicher Ebene verbleiben;

- Rücksichtnahme auf die materielle und immaterielle Ressourcenausstattung, um Überforderungstendenzen vorzubeugen und eine fortbestehende Kooperationsdividende sicherzustellen.

Im Grundsatz sind diese Kriterien dem europäischen Integrationsprozess nicht fremd, wie etwa die Verankerung des bislang freilich so gut wie nie verfolgten Subsidiaritätsprinzips (vgl. u.a. in der Präambel des Vertrags über die Europäische Union) verdeutlicht. ${ }^{11}$ Gleichwohl dokumentiert ein Blick auf den gegenwärtigen Aufgabenzuschnitt der Union, dass in mehreren Dimensionen (etwa mit Blick auf die einbezogenen Politikbereiche, die dabei gewählte Regelungs- und Verwaltungstiefe sowie den verfügbaren Ressourcenrahmen) deutliche Entwicklungspotentiale, in Teilen aber auch Rückzugstendenzen (aufgrund nicht eingelöster Ansprüche oder wirkungsloser Politiken) erkennbar sind.

Eine den Namen verdienende Aufgabenanalyse verfolgt das Ziel, solche Berei-

11 Vgl. hierzu auch den Beitrag von Peter M. Huber in diesem Heft. 
che zu identifizieren und ggf. Korrekturvorschläge zu unterbreiten. Das Interesse richtet sich dabei vor allem auf die gebietskörperschaftliche Verortung der Aufgabenwahrnehmung (Vollzugskritik), weniger auf die Frage nach der politischen Notwendigkeit einer bestimmten Aufgabe oder deren konkreter Umsetzung (Zweckkritik). Unter Beachtung der angesprochenen Kriterien und ohne etwaige issue linkages oder andere Verflechtungsprozesse zu vernachlässigen, ist der gegenwärtige Aufgabenbestand dabei zu überprüfen und gegebenenfalls mit einer von drei Handlungsempfehlungen zu versehen: Ein etwaiger Aufgabenverzicht bietet sich für jene Bereiche an, die erkennbar die institutionelle, prozessuale und ressourcenbezogene Tragfähigkeit der Union überfordern. Im Gegensatz dazu bietet sich eine Aufgabenkonzentration dann an, wenn ein grenzüberschreitendes Politikfeld sich für die europäischen Einrichtungen und Verfahren als besonders geeignet erweist und daher ggf. einer erweiterten Europäisierung unterliegen sollte. Schließlich besteht die Möglichkeit, durch Flexibilisierung und Koordinierung der Aufgabenwahrnehmung Mischformen und arbeitsteilige Verfahren einzusetzen, was durchaus der jüngeren Staatspraxis im Rahmen europäischer Politik entspricht.

\section{Aufgabenverzicht}

Mehr noch als etwaige Ergänzungen stellt der Verzicht auf politikbereichspezifische Kompetenzen zugunsten einer nachgeordneten gebietskörperschaftlichen Ebene stets ein eher in Ausnahmefällen anwendbares Vorgehen dar. Die Gründe hierfür liegen neben den oft nur schwer abschätzbaren Anpassungskosten (auch für die jeweilige Klientel) vor allem in der institutionellen Reformunwilligkeit der bisher mit den entsprechenden Aufgaben betrauten Einrichtungen, deren Akteure sich um den eigenen Einfluss und vormals genossene Sach- wie Finanzmittel sorgen. Auch am anderen Ende des Transferprozesses darf nicht davon ausgegangen werden, dass der Aufgabenzuwachs unbedingt begrüßt wird. Häufig erscheinen die dort geschaffenen Kapazitäten als unzureichend und entsteht somit, in der Wahrnehmung vieler Beteiligter, eine gewisse Zusatzbelastung. Aus diesem Grund müssen erkennbare Aufgabenüberschüsse deutlich prononciert ausfallen, um das für Reformen notwendige Momentum zu generieren. Darunter fallen jene Aufgaben, deren Zuordnung zur europäischen Ebene aufgrund der begrenzten Aufgabentragfähigkeit der Union mehr Probleme schafft als auflöst. Solche Aufgaben binden Ressourcen, die ggf. dezentral besser verteilt und über deren Verwendung auf mitgliedstaatlicher oder gar regionaler Ebene effizienter entschieden und verfügt werden könnte. 
Legt man die bislang für europäische Aufgaben entwickelten Kriterien (Vollzugsferne, Regulierungs-/Harmonisierungsfähigkeit und Ressourcenschonung) zugrunde, fallen im Rahmen der Union insbesondere jene Politikbereiche ins Auge, deren Bearbeitung durch eigene Exekutivorgane vorgesehen ist, etwa über das Institut europäischer Agenturen und sog. An-Behörden. Die Befassung nachgeordneter Verwaltungseinrichtungen widerspricht den drei benannten Tragfähigkeitskriterien in besonderem Maße; auch das geringe politisch-administrative Profil der damit angesprochenen Einrichtungen (bekannt als quasi nongovernmental organisations oder quangos ${ }^{12}$ ) könnte eine gewisse Überbetonung des Politikbereichs bewirken. Der liberale think tank Open Europe zählte im vergangenen Jahr 52 solcher Einrichtungen, die jährliche Gesamtkosten in Höhe von 2,64 Mrd. EUR verursachten. Die Zahl der Einrichtungen hat sich seit der Jahrtausendwende mehr als verdreifacht, wobei häufig Parallelstrukturen erkennbar sind und Aufgaben bearbeitet werden, die in ähnlicher oder gleicher Form bereits von den Organen der EU (vorrangig der Kommission) oder anderen quangos wahrgenommen werden (sollten). Wie eine von der Kommission selbst in Auftrag gegebene Beurteilung schlussfolgert, schaffen diese Agenturen und Behörden , an indirect but powerful incentive for spending “. ${ }^{13}$ Während etwa die Grenzschutzagentur FRONTEX oder die Chemiebehörde ECHA im Rahmen des Binnenmarktes erkennbar notwendige Koordinationsaufgaben im Auftrag der Kommission und der Mitgliedstaaten erfüllen, verweisen viele dieser Einrichtungen auf deutliche Aufgabenüberschüsse. Sie lassen sich in zwei Gruppen einteilen.

Zum einen geht es um doppelte Zuständigkeiten und die sich damit verbindende Ineffizienz wie den erkennbaren Ressourcenverschleiß. Als Beispiele können etwa die Bereiche Menschenrechtspolitik und Arbeitsmarktpolitik gelten. In Ersterem agiert u.a. die Agentur der Europäischen Union für Grundrechte (EAFR), deren Budget sich auf etwa 20 Mio. EUR beläuft. Davon abgesehen, dass der Grundrechts- und Menschenrechtsschutz zwar zu den fundamentalen Grundsätzen der Union zählt, allerdings kein eigenes Politikfeld darstellt, erscheint die Tätigkeit dieser aus einer Aufwertung der früheren Antidiskriminierungsstelle entstandenen Agentur höchst fragwürdig. So dupliziert sie nicht nur die Arbeit existierender Behörden in den meisten Mitgliedstaaten, sondern steht auch in direkter Sachkonkurrenz zu den Kommissaren für Justiz und Grund-

12 Open Europe: The Rise of the EU Quangos, Updated Version, London, 2012.

13 Ebd., 2. 
rechtsfragen sowie deren Generaldirektoraten. Hinzu tritt, dass mit dem Europarat und dem Europäischen Gerichtshof für Menschenrechte bereits wirksame und großzügig ausgestattete europäische Einrichtungen außerhalb der Union bestehen, die sich ausführlich mit der Themenstellung befassen.

Im Bereich der Arbeitsmarktpolitik, der ohnehin nur in engen Grenzen in die Zuständigkeit der Union fällt, stechen gleich vier solcher Einrichtungen hervor: die Europäische Stiftung für Berufsbildung (ETF), das Europäische Zentrum für die Förderung der Berufsbildung (CEDEFOB), die Europäische Stiftung zur Verbesserung der Lebens- und Arbeitsbedingungen (EUROFUND) sowie die Europäische Agentur für Sicherheit und Gesundheitsschutz am Arbeitsplatz (EUROFOUND). Jenseits der auch hier fraglichen Zuständigkeit muss festgestellt werden, dass sich die Einrichtungen in ihrem konkreten Aufgabenzuschnitt überlappen und dabei erkennbar die seitens der Kommission verfolgten Arbeiten zum Themenbereich Arbeitsplatzsicherheit, Berufs- und Fortbildung sowie Arbeitsschutz spiegeln. Hinzu tritt, dass aufgrund europarechtlicher Regelungen (etwa zum Arbeitnehmerschutz) auch auf nationalstaatlicher Ebene entsprechende Einrichtungen gestärkt oder geschaffen wurden. Zudem wird der Politikbereich, wie im Fall der Grundrechtspolitik, stark von den pluralistischen Interessenvertretungen der Zivilgesellschaft bearbeitet, auf europäischer wie nationalstaatlicher Ebene.

Zum anderen richten sich die Bedenken auf Einrichtungen, die Aufgaben erfüllen, deren Wahrnehmung auf europäischer Ebene im Allgemeinen eher fragwürdig erscheint. Ein Beispiel hierfür ist die Exekutivagentur Bildung, Audiovisuelles und Kultur (EACE), die eigens zur Verwaltung ,,bestimmter Teile der EUProgramme in den Bereichen Bildung, Kultur und audiovisuelle Medien“ geschaffen wurde. In Verbindung mit dem zuständigen Generaldirektorat sind in diesem Politikbereich annähernd doppelt so viele Menschen beschäftigt als im wesentlich bedeutsameren Generaldirektorat für den Binnenmarkt (mehr als 850 Bedienstete im Bereich Kultur gegenüber etwa 450 Bediensteten im Binnenmarkt- und Dienstleistungs-Generaldirektorat).

All dies verweist bereits exemplarisch auf deutliche Rückführungspotentiale, sei es vom Verwaltungsunterbau hin zur Kommission, sei es von der europäischen Ebene hin (besser: zurück) zu den Nationalstaaten. Addiert man dem die wachsenden Zweifel etwa an der wirtschaftlichen wie ökologischen Sinnhaftigkeit einer einheitlich verwalteten europäischen Landwirtschafts- und Fischereipolitik oder dem micro management immer weiterer binnenmarktbezogener Politikfelder (von der Sozialpolitik bis hin zum europaweiten Verbot ganzer Produktkate- 
gorien, wie etwa der traditionellen Glühbirne), so wird der Bedarf an einer intensivierten oder sich ggf. verstetigenden Aufgabenkritik auf europäischer Ebene deutlich. Dies gilt umso mehr, als in einigen Politikfeldern auch eine Aufgabenkonzentration denkbar ist, mithin ein noch verstärktes Interesse an der Vermeidung unnötiger Belastungen und offensichtlichen Ressourcenverschleißes besteht.

\section{Aufgabenkonzentration}

Neben einer möglichen Rückführung von Aufgaben auf die mitgliedstaatliche Ebene - vor allem in Vollzugsfragen - findet sich in einigen Bereichen auch ein Bedarf, gegebene Aufgaben der europäischen Ebene sinnvoll zu ergänzen oder solche Politikbereiche, die auf mitgliedstaatlicher Ebene erkennbar zu kleinteilig wahrgenommen werden, zumindest in Teilen in die Zuständigkeit der Union zu überführen. Auf der Grundlage dieser Unterscheidung sollen nachfolgend einige Politikfelder exemplarisch skizziert werden, die sich für eine stärkere Aufgabenkonzentration eignen. Die funktional begründete Ergänzung und Ausweitung des bestehenden Aufgabenprofils gilt vielen als einer der wesentlichsten Gründe für die Entwicklung des Europäisierungsprozesses in den vergangenen 60 Jahren. Und tatsächlich weist Einiges darauf hin, dass ein gewisser functional spill-over zur Vergemeinschaftung von Politikbereichen beigetragen hat. Gleichwohl stößt diese Logik vor allem dann an ihre Grenzen, wenn Bereiche tangiert werden, die einige Mitgliedstaaten als besonders sensibel wahrnehmen, was wiederum zu einer Mobilisierung von Vetopotentialen beiträgt.

So verfügt die Europäische Union weiterhin über keine eigene fiskalische Basis, agiert sie nach wie vor uneinheitlich mit Blick auf ihre geoökonomische wie geopolitische Position gegenüber Drittstaaten und finden sich trotz aller Harmonisierungsbestrebungen vielfältige Parallelstrukturen auf europäischer und nationalstaatlicher Ebene. Bestrebungen zur weiteren Öffnung des Binnenmarktes werden durch kontraproduktive nationale Anreizwettbewerbe, insbesondere im Bereich der Kapitaleinkünfte- und Unternehmensbesteuerung, ins Negative verkehrt, während sich in zahlreichen anderen Politikbereichen Potential zur Harmonisierung und Koordination mitgliedstaatlicher Politiken findet. Auch im Bereich der Wirtschaftsunion steht weiterhin die Einführung einer einheitlichen Finanzmarkt- und Bankenregulierung aus.

Legt man auch hier die angesprochenen Kriterien Vollzugsferne, Regulierungs-/ Harmonisierungsfähigkeit und Ressourcenschonung zugrunde, kann die Notwendigkeit verstärkter Aufgabenkonzentration etwa im Bereich der Entwick- 
lungspolitik verdeutlicht werden, da hier beide angesprochenen Dimensionen zum Tragen kommen: die sinnvolle Ergänzung bestehender Aufgaben bei funktional fragwürdiger Kleinteiligkeit der nationalstaatlichen Aufgabenwahrnehmung. So spricht bereits der langjährige Konflikt zwischen außen- und sicherheitspolitischen Interessen auf der einen Seite und den Zielen einer nachhaltigen Entwicklungspolitik auf der anderen für eine verstärkte Europäisierung des Politikfeldes. Nationalstaatliche Entwicklungsorganisationen stehen stets im Spannungsfeld zwischen Eigeninteresse und objektivem Handlungsbedarf, wie etwa die langjährige Diskussion um eine Zusammenführung von Auswärtigem Amt und Entwicklungsministerium in Deutschland verdeutlicht. Da die Kernthemen der Außen- und Sicherheitspolitik auf absehbare Zeit weiter dem Primat nationalstaatlicher Politik unterliegen werden, spricht allein diese Spannungskonstellation für eine Entflechtung der Entwicklungspolitik durch europäisierte Koordination von Zielen und Mittelvergabe. Hinzu tritt, dass die Union durch ihre Alleinzuständigkeit in Fragen der Handelspolitik bereits über ein äußerst entwicklungsrelevantes Instrumentarium verfügt, etwa um präferentielle Marktzugangsregeln oder Zollerleichterungen zu ermöglichen. Schließlich könnte eine umfassende gemeinschaftliche Entwicklungspolitik, die über die gegenwärtige „,ergänzende“ Funktion hinauswächst, zur Auflösung des internen Zielkonflikts zwischen marktliberaler Handelspolitik und protektionistischer Agrarpolitik beitragen.

Zurzeit verausgabt die Union im Bereich der Entwicklungspolitik jährlich etwa 12,6 Mrd. USD (Stand: 2011), während die größten EU-Geberländer (Mitglieder im Entwicklungsausschuss der OECD) im gleichen Zeitraum gemeinsam etwa 72,3 Mrd. USD an offiziellen Hilfeleistungen zur Verfügung stellen. Die mitgliedstaatlichen Programme folgen je eigenen Zielsetzungen und finden sich nicht selten in Wettbewerbs- oder Widerspruchssituationen zueinander. Zwar ist eine gewisse territoriale Arbeitsteilung entlang der traditionellen post-kolonialen Verbindungslinien erkennbar, doch kann in diesem Bereich gleichwohl von einer deutlich ausbaufähigen Kapazität zur Koordination von Zielformulierung und Programmentwicklung ausgegangen werden. Die nationalstaatlichen Agenturen würden, bei Schaffung kostensparender zentraler Dienste, als Vollzugseinrichtungen weiterbestehen, während die Leitungsebene (also die jeweils obersten Organe, meist Ministerien) drastisch reduziert werden könnten. Über den Weg einer verstärkt koordinierten und auf einheitliche Zielsetzungen verpflichteten europäischen Entwicklungspolitik würde zudem das auswärtige Handeln der Union aufgewertet, ohne einer eher unrealistischen umfänglichen Zusammenar- 
beit im Bereich der Außen- und Sicherheitspolitik zu harren. Schließlich käme eine europäische Entwicklungspolitik dem Konzept einer normativ ausgerichteten und über eher weiche Formen der Diplomatie wirkenden Europäischen Union entgegen.

Weitere Beispiele ließen sich etwa für den Bereich der Außengrenzen formulieren, der eine gemeinsame Flüchtlings- und Asylpolitik immer dringlicher erscheinen lässt, oder für die gegenwärtig ubiquitär diskutierte Frage nach einer erweiterten wirtschaftspolitischen Koordination im Rahmen der Währungsunion (Bankenunion, Fiskalunion etc.). Eine Aufgabenkonzentration ist dabei im Allgemeinen für all jene Bereiche zu erwägen, die europäischer Standardsetzung bedürfen oder von ihr profitieren könnten, wobei demokratischen Vorbehalten sowie vor allem etwaigen Pfadabhängigkeiten Rechnung getragen werden muss, schon um allzu hohen Ausstiegskosten vorzubeugen. Im Zentrum stünden vor allem die Nutzung des derzeit rechtlich verfügbaren Aufgabenportfolios, die freiwillige Aufgabenübertragung durch die Mitgliedstaaten sowie kleinere Vertragsanpassungen und passerelle-Lösungen. Deshalb bietet sich vor allem die Konzentration auf ,,schlanke“ institutionelle Lösungen an, deren Fokus auf den Bereichen Information, Kommunikation, Willensbildung, Politikformulierung und Entscheidung liegen sollte, während die Mitgliedstaaten - aufgrund positiver Routinen und berechtigter Absicherungsinteressen - weiterhin für den konkreten Vollzug verantwortlich zeichnen sollten.

\section{Flexibilisierung und Koordinierung}

Während die angesprochenen Formen von Aufgabenverzicht und Aufgabenkonzentration auch bei strenger Ausrichtung an den angesprochenen Kriterien mit einer Reihe von rechtlichen, institutionellen und ressourcenbezogenen Hürden verbunden wären, trifft dies für die dritte und im Fall der Europäischen Union wohl bedeutsamste Form der Aufgabenanpassung nur begrenzt zu: die Flexibilisierung der Aufgabenwahrnehmung und die institutionalisierte Koordinierung von Zielvorstellungen und Umsetzungsvorgaben durch die handelnden Akteure. Diese Form der Anpassung eignet sich somit insbesondere für jene breite Grauzone zwischen genuin subsidiär wahrzunehmenden Aufgaben und erkennbar eines europäischen Gemeinschaftsansatzes bedürfenden Politikfeldern. Flexibilisierte Ansätze und verstärkte Koordination würden in doppelter Hinsicht Übergangs- und Teillösungen ermöglichen: zum einen durch eine partielle Europäisierung von Politikbereichen, zum anderen durch die Verständigung auf eine 
Aufgabenverschiebung im Rahmen einer Teilgruppe von Mitgliedstaaten (sog. Club-Lösungen).

Beide Formen der Aufgabenflexibilisierung sind im europäischen Kontext höchst umstritten. Verfechtern wird schnell vorgeworfen, sie betrieben eine Spaltung des Kontinents, förderten ein Europa unterschiedlicher Geschwindigkeiten oder redeten einer à-la-carte-Union das Wort. Zugleich hat die realpolitische Notwendigkeit derartiger Teillösungen sich bereits vor mehr als 20 Jahren mit den Beschlüssen zur Währungsunion, zum Maastrichter Sozialprotokoll und zum Schengen-Raum bewährt und durchgesetzt; über die Instrumente der (unverbindlichen und eher wenig erfolgreichen) Offenen Methode der Koordinierung sowie der 1997 eingeführten Verstärkten Zusammenarbeit wurde ein solches Vorgehen zudem für faktisch alle Politikbereiche geöffnet. ${ }^{14}$ Die Verstärkte Zusammenarbeit wurde 2010 im Rahmen einer von 14 Mitgliedstaaten konzertierten Reform des Scheidungsrechts erstmals angewandt, Anfang 2013 folgte eine Einigung auf ein einheitliches Patentrecht. Die Verstärkte Zusammenarbeit wird zwar stets als Mittel der letzten Wahl gekennzeichnet und sieht sich vergleichsweise hohen Anforderungen ausgesetzt (etwa: mindestens neun Mitgliedstaaten müssen einen Vorschlag ab ovo unterstützen), doch stellt sie gleichwohl eine Verstetigung des hier angesprochenen Umgangs mit Fragen der Aufgabenwahrnehmung dar.

Flexibilisierung und Koordinierung sind vor allem dann vorrangig anwendbar, wenn weder eine notwendige Aufgabenergänzung noch eine ernsthafte Aufgabenrückführung möglich erscheinen, etwa aufgrund des Widerstands Einzelner oder bestehender Pfadabhängigkeiten. Zudem stellen beide Vorgehensweisen sinnvolle Ergänzungen nationalstaatlicher Politik dar, indem die Stärke der Union in den Bereichen Informationsabgleich und Standardevaluation eng mit dem Vollzugspotential der Mitgliedstaaten verbunden bleibt: Die federführenden Einrichtungen sind hier stets mitgliedstaatliche Regierungsorgane, nicht etwa die Europäische Kommission. Schließlich bieten sie sich den Mitgliedstaaten als Möglichkeit zur Überwindung politischer oder verfassungsrechtlicher Beschränkungen einer weiteren Vergemeinschaftung von Aufgaben an, indem das Ausweichen auf die intergouvernementale, ggf. völkerrechtliche Ebene ermöglicht wird. Letzteres dürfte in Deutschland insbesondere mit Blick auf die LissabonRechtsprechung des Bundesverfassungsgerichts von Bedeutung sein, betrifft aber

14 Hesse, J.J./Grotz, F.: Europa professionalisieren. Kompetenzordnung und institutionelle Reform im Rahmen der Europäischen Union, Berlin, 2005; dies.: „Flexibilisierung“ europäischer Politik als Weg aus der Krise, in: Häberle, P. (Hg.): Jahrbuch des öffentlichen Rechts, 54 (2006), 607-628. 
auch traditionell eher ,euroskeptische“ Mitgliedstaaten, wie etwa das Vereinigte Königreich.

Die Benennung geeigneter Aufgaben- und Handlungsfelder entfällt in diesem Fall, da das Ziel einer verstärkten Flexibilisierung und einer erweiterten Koordinierung gerade nicht in ex ante festgeschriebenen Vorgaben besteht. Vielmehr geht es darum, die Aufgabenwahrnehmung im Bedarfsfall zu ermöglichen, ohne sie bei abklingender Notwendigkeit zu erzwingen. Dadurch werden formalisierte Aufgabenkonzentrations- und -rückführungsprozesse, die stets mit hohen Anpassungskosten verbunden sind, vermieden, ohne die Handlungsfähigkeit der Union einzuschränken. Insofern stellt diese Form der Aufgabenanpassung in Theorie und Praxis die derzeit vielversprechendste Möglichkeit dar, das Aufgabenspektrum der Europäischen Union an gegenwärtige und künftige Herausforderungen anzupassen.

\section{Handlungsoptionen einer Aufgabenanpassung}

Die angesprochenen Formen der Aufgabenentwicklung auf europäischer Ebene orientierten sich zwar gesamthaft an den Kriterien Vollzugsferne, Regulierungs-/ Harmonisierungsfähigkeit und Ressourcenschonung, doch sind durchaus unterschiedliche Ansätze zur Umsetzung einer solchen Aufgabenanpassung denkbar. Nachfolgend werden drei von kleinteiligen Lösungen bis hin zu großflächigen bzw. übergreifenden Reformen reichende (skalierte) Handlungsmöglichkeiten umrissen, die sich mit einer abnehmenden Umsetzungswahrscheinlichkeit verbinden:

- die Optimierung des Status quo,

- $\quad$ eine Aufwertung der Verstärkten Zusammenarbeit sowie

- $\quad$ eine umfassende Funktionalreform.

Diese Möglichkeiten werden mit Blick auf aktuelle und künftig erwartbare Entwicklungen skizziert und hinsichtlich erkennbarer Hürden evaluiert. Hinzu tritt die ergänzende Möglichkeit einer Form der verstetigten Aufgabenanalyse und Aufgabenkritik im Rahmen einer ,Strukturberichterstattung für die Gebietskörperschaften“ ${ }^{15}$, die ggf. auch unabhängig oder in Vorbereitung der benannten Handlungsoptionen verfolgt werden sollte.

15 Hesse, J.J.: Strukturberichterstattung für die deutschen Gebietskörperschaften: Methodische Vorstudien, Berlin, 2008. 


\section{Optimierung des Status quo}

Eine Optimierung des Status quo setzt die grundsätzliche Anerkennung der gegebenen Aufgabenstruktur voraus und sucht nach den Erfahrungen mit dem Verfassungsvertrag und dem Vertrag von Lissabon vor allem eine weitere ,groBe" Vertragsrevision zu vermeiden. Dabei stehen punktuelle Veränderungen in offensichtlich dysfunktionalen Bereichen im Vordergrund.

Das jüngste Beispiel für diese Form der Entwicklung stellt das muddling through im Rahmen der Wettbewerbsfähigkeits- und Verschuldungskrise dar: Schaffung von privatrechtlichen Instrumenten zur Bündelung der Finanzhilfen; stillschweigende Flexibilisierung der Regeln zur Staatsfinanzierung; Nutzung der gemeinschaftsrechtlichen Gesetzgebungskompetenzen, um offensichtliche Regelungslücken zu füllen und Fehlentwicklungen zu korrigieren (vgl. die six-pack- und twopack-Gesetzgebung); nötigenfalls Auslagerung bedeutsamer Regelungen in intergouvernementale/völkerrechtliche Verträge (ESM-Vertrag, Fiskalpakt); sowie schließlich die sehr gering dosierte und auf das nötigste beschränkte Veränderung der Vertragsgrundlagen, möglichst ohne Notwendigkeit eines Referendums (Ergänzung des AEU-Vertrags um einen ESM-Passus).

Die Vorteile eines solchen Vorgehens liegen auf der Hand: Neben der Nutzung der bereits gegebenen Spielräume wird vor allem darauf geachtet, die unmittelbaren politischen und wirtschaftlichen Kosten zu minimieren, indem man auf umfangreiche Vertragsrevisionen verzichtet und nötigenfalls Nebenabsprachen trifft. Abgesehen von Krisenreaktionsmaßnahmen ist ein solches Vorgehen unter anderem bei der Reform etablierter Aufgabenfelder denkbar, etwa im Rahmen einer Rückführung von Überschussenergien in den Bereichen Binnenmarkt und Außenpolitik oder im Rahmen einer ggf. auch nachträglichen (und ernsthaften) Normenkontrolle und -überprüfung. ${ }^{16}$ Zugleich ist aber auch auf die eher begrenzte materielle wie zeitliche Reichweite einer solchen Optimierung zu verweisen: Während der rechtliche Rahmen die erreichbaren Veränderungen begrenzt, sind einfach umzusetzende Lösungen auch ebenso einfach zu revidieren, was ggf. im Rahmen einer Betrachtung der Anreizstrukturen politisch Handelnder zu Kurzfristdenken beitragen könnte. Schließlich wird gerade im Rahmen der EuroRettungspolitik deutlich, dass auch ohne eine umfassende Anpassung der

16 Die dafür in zahlreichen EU-Mitgliedstaaten eingesetzten Normenkontrollräte sind in ihrer Reichweite sehr unterschiedlich einzuschätzen. Der in den deutschen Gesetzgebungsprozess eingebundene Normenkontrollrat gilt bislang als wenig erfolgreich, zumindest blieb der Ertrag weit unter den bei seiner Einrichtung formulierten Erwartungen. 
Rechtsgrundlagen horrende Haftungs- und Zahlungsverpflichtungen nicht nur für die Gegenwart, sondern vor allem auch für die Zukunft eingegangen werden können. Es bleibt mithin zu diskutieren, ob und inwiefern ein solch niederschwelliges Handeln in allen Kontexten tatsächlich empfehlenswert ist.

\section{Aufwertung der Verstärkten Zusammenarbeit}

Die Aufwertung des Instruments der Verstärkten Zusammenarbeit setzt hingegen die Bereitschaft voraus, diese Form der Kooperation und Koordination über eine begrenzte Vertragsrevision zu einem regulären intergouvernementalen Verfahren der Union zu erklären und die Kommission darauf zu verpflichten, solche Vorhaben, auch wenn sie von den Mitgliedstaaten ausgehen, nach Kräften zu unterstützen. Im Fall des langjährigen Streits um ein europäisches Patentrecht war es die Kommission, die auf diesem Weg - im Einvernehmen mit 25 Mitgliedstaaten - ein Gesetzgebungsvorhaben zu ,retten“ versuchte, das von Spanien und Italien nachhaltig blockiert wurde. Gegebenenfalls ist im Rahmen einer solchen Revision auch das Genehmigungsverfahren für eine Verstärkte Zusammenarbeit zu überprüfen, um die Erfordernis einer Qualifizierten Mehrheit umzukehren: Während die Mehrheit zurzeit für die Genehmigung stimmen muss, könnte stattdessen über eine Vetomehrheit nachgedacht werden. Dies würde es auch Gruppen von kleineren Mitgliedstaaten erlauben, eine Verstärkte Zusammenarbeit zu initiieren. Schließlich sollte erwogen werden, eine cooperation renforcée auch mit Blick auf mitgliedstaatlich wirksame und vollzugsrelevante Aspekte jener Politikfelder zuzulassen, die in der ausschließlichen Zuständigkeit der Union liegen. Hierbei wäre allerdings auf eine strikte Überwachung durch die Kommission und den EuGH Wert zu legen.

Die Frage der Aufgabenverteilung wäre bei dieser Anpassung kein unmittelbarer Verhandlungsgegenstand, was angesichts der anderenfalls erwartbaren Reformhürden als förderlich gelten kann. Ein positiver Nebeneffekt fände sich zudem in dem entstehenden Verfahrenswettbewerb zwischen Gemeinschaftsmethode, intergouvernementaler Einstimmigkeit und Verstärkter Zusammenarbeit von Teilgruppen (und in Teilbereichen). Die Anreize würden zugunsten einer erweiterten Handlungsfähigkeit und zuungunsten des Status quo verschoben, während bestehende Aufgaben weiterhin ungehindert ausgeführt werden können, die Kooperationsrenditen der nicht beteiligten Mitgliedstaaten mithin kaum berührt wären. 
Auch diese Handlungsoption zeichnet sich durch eher geringe Kosten aus und dürfte in vielen Fällen mindestens eine second-best-Variante darstellen. Zugleich sollten auch die potentiellen Nachteile eines erschwerten (da mit erhöhten informatorischen und koordinativen Anforderungen konfrontierten) Vollzugs sowie der Schaffung von ggf. später schwer überwindbaren Teilungen und Ausdifferenzierungen innerhalb der Union nicht außer Acht gelassen werden. Insbesondere in Fällen einer Aufspaltung der Mitgliedstaaten in zwei sich entgegenstehende Lager könnte es schnell zu konkurrierenden Lösungsansätzen kommen, die zwar kurzfristig Probleme beheben, langfristig aber eher zur Schaffung tiefgreifenderer Verwerfungen und erkennbarer Systemrivalitäten beitragen. Zur Vermeidung einer solchen Situation wären daher die Mehrheitserfordernisse für die Einleitung einer Verstärkten Zusammenarbeit entsprechend anzupassen.

\section{Funktionalreform}

Eine Funktionalreform schließlich stellt die aufwendigste Handlungsoption dar, ${ }^{17}$ vor allem aufgrund der umfassenden Primärrechtsanpassungen, die sich mit einem solchen Vorhaben verbinden. Sie setzt mithin die Bereitschaft der beteiligten Akteure zu einer grundlegenden Überprüfung und Anpassung des Aufgabenzuschnitts der Europäischen Union voraus, wie sie etwa der britische Premier Cameron unlängst einzufordern schien. Es ist schwer vorstellbar, dass eine Reform dieses Ausmaßes ohne die Einberufung eines weiteren Konvents vonstatten gehen könnte. In diesem Fall wäre auch mit Blick auf die Konventsarbeit eine umfassende Überprüfung der bisherigen Routinen erforderlich, um die im Rahmen des Verfassungskonvents erkennbaren Tendenzen hin zu einer gewissen Verselbstständigung und $\mathrm{zu}$ einer möglichst breiten Auftragsinterpretation zu vermeiden. $\mathrm{Ob}$ mit oder ohne Konvent ${ }^{18}$ - eine solche Reform wäre sachlich daran gehalten, eine umfassende Aufgabentragfähigkeitsanalyse der europäischen Ebene vorzunehmen, um in einem zweiten Schritt die gegenwärtige funktionale Ausrichtung zu überprüfen und ggf. zu verengen oder zu ergänzen. Schließlich müssten die strukturellen Gegebenheiten nötigenfalls angepasst werden, wobei an dieser Stelle deutlich für eine gesamthafte Funktionalreform plädiert wird. Institutionelle, kompetenz- und ressourcenbezogene Anpassungen

17 Hesse, J.J.: Aufgabenkritik, Funktional- und Strukturreform, a.a.O.

$18 \mathrm{Zu}$ einer ernüchternden Analyse der Handlungsmöglichkeiten durch einen Konvent vgl. Hesse, J.J.: Vom Werden Europas. Der EU Verfassungskonvent - Auftrag, Ansatz, Ergebnisse, Berlin, 2007. 
bildeten demnach zwar konstitutive Elemente, wären zunächst aber von sekundärer Bedeutung.

Folgt man auch hier den Kriterien Vollzugsferne, Regulierungs-/Harmonisierungsfähigkeit und Ressourcenschonung, so wären insbesondere die Bereiche Fischerei- und Landwirtschaftspolitik nachhaltig zu überprüfen, während der gesamte Binnenmarktbereich, v.a. mit Blick auf die Regulierung von Banken und Finanzmärkten, sowie das Feld der Währungspolitik einer erweiterten europäisierten Steuerung bedarf. Sucht man ein solches Vorgehen systematisch darzustellen, wäre aufgrund der bestehenden Aufgabenstruktur eine Neu-Zuordnung entlang der erkennbaren Handlungsebenen vorzunehmen. Zunächst müsste das europäische Aufgabensystem einer gewissen Vereinfachung unterliegen, indem zwischen drei Kompetenzformen unterschieden wird (vgl. Tab. 1): alleiniger EUZuständigkeit (europäische Pflichtaufgaben), konkurrierender EU-Zuständigkeit (freiwillige Aufgabenübernahme durch die EU bei komplementärer Aufgabenabgabe der Mitgliedstaaten) sowie zwischenstaatlichen Aufgaben. Die kategoriale Unterscheidung zwischen mitgliedstaatlichen Aufgaben, die europäischen koordinierenden Initiativen offenstehen, und solchen, die ggf. als Ergebnis mitgliedstaatlicher Anregung durch die EU koordinierend oder ergänzend begleitet werden können, wäre damit zugunsten der Mitgliedstaaten aufgehoben. Für ihren Aufgabenkreis wären sie vollumfänglich in der Lage, den Grad intergouvernemental-europäischer Koordination zu bestimmen (bestenfalls bei zugleich etablierter Möglichkeit einer Verstärkten Zusammenarbeit von Teilgruppen).

Der große Vorteil dieser Alternative liegt in der umfassenden Betrachtung der Aufgabentragfähigkeit der Union, die bestenfalls sämtliche Politikfelder betrifft und keine vorab definierten Schutzbereiche kennt. Dies ermöglicht zum einen die gesamthafte Betrachtung dessen, was die Union gegenwärtig zu leisten vermag, zum anderen eine Herausarbeitung jener Aufgaben, die auf mitgliedstaatlicher Ebene besser, effektiver und effizienter erledigt werden können. Dieser unsentimentale Blick auf Sinn und Zweck mancher Unionszuständigkeiten würde ergänzt durch eine Konzentration einiger zusätzlicher Aufgaben auf europäischer Ebene - mit der Aussicht auf eine mittel- und langfristig tragfähige Aufgabenverteilung. Aufgrund der enormen Konsens- und Anpassungskosten dieses Vorgehens ist damit jedoch kaum zu rechnen; schon die Verpflichtung aller Mitgliedstaaten auf ein solches Vorhaben dürfte nur schwer zu verwirklichen sein. Und selbst wenn dies gelingen sollte, erscheint eine konsequente und zielführende Umsetzung angesichts vorliegender Erfahrungen mit ähnlichen Vorhaben weitgehend illusorisch. 
Tabelle 1: Exemplarische Darstellung der Auswirkungen einer tragfähigkeitsgestützten Funktionalreform auf die europäische Aufgabensystematik

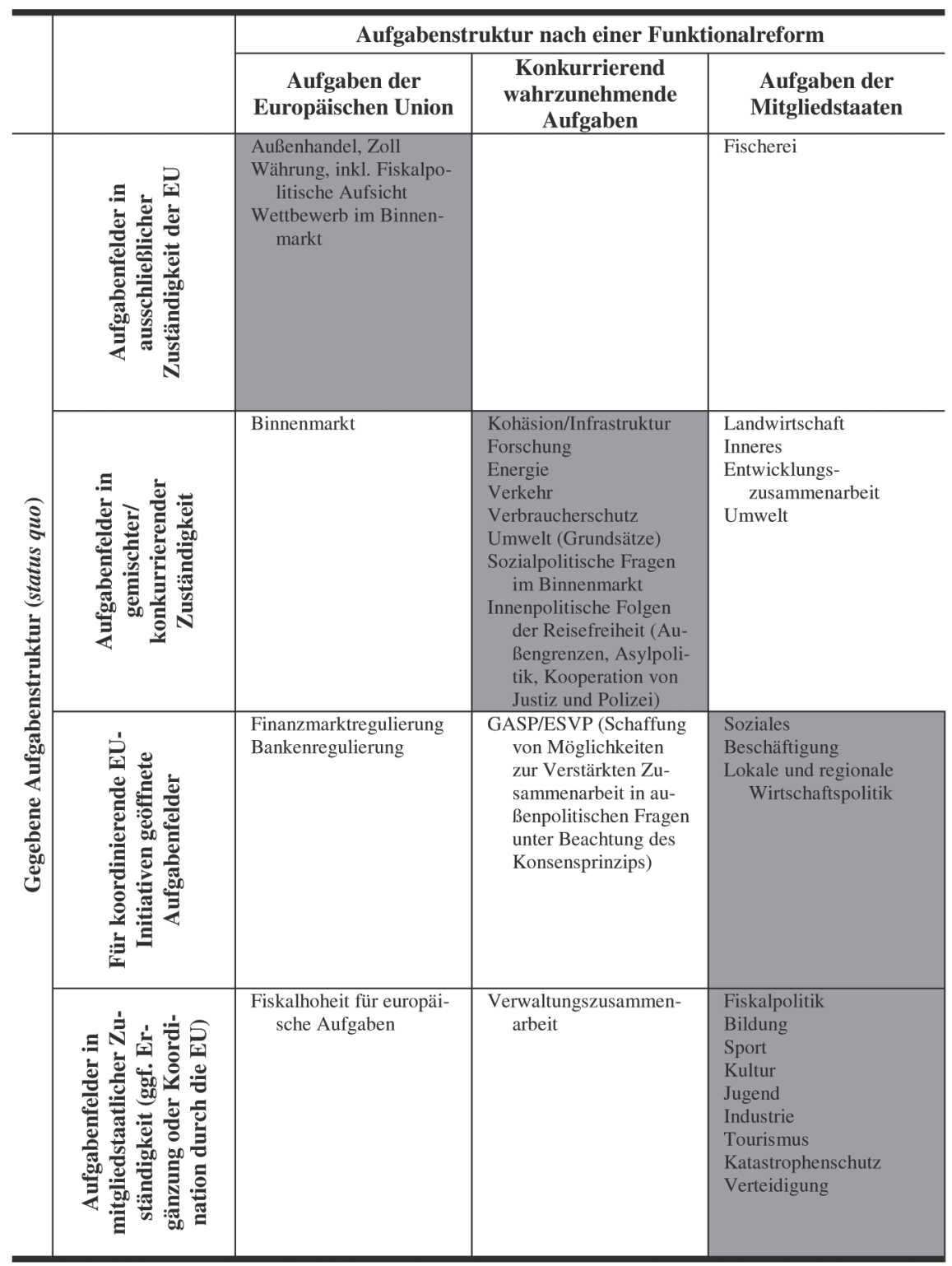

Anmerkung: Die grau hinterlegten Felder kennzeichnen gegenüber dem Status quo unveränderte Zuständigkeiten. Quelle: Eigene Darstellung nach der Strukturberichterstattung für die deutschen Gebietskörperschaften, ISE Berlin, 2013. 


\section{Kontinuierliches Monitoring und Vergleich institutioneller Vorausset- zungen}

In Ergänzung der gewählten Handlungsoption erscheint schließlich die Einrichtung eines verstetigten monitoring samt eines den Namen verdienenden Vergleichs institutioneller Voraussetzungen auf europäischer wie nationalstaatlicher Ebene angezeigt. Eine punktuelle Analyse der Aufgabentragfähigkeit und angemessenheit kann nicht zur dauerhaften Lösung der Probleme des öffentlichen Handelns auf den unterschiedlichen gebietskörperschaftlichen Ebenen beitragen, auch sollte die EU bei einer solchen Überprüfung natürlich nicht ausgeschlossen bleiben. Zugleich ist keine unabhängige Einrichtung auf europäischer Ebene erkennbar, die diese Aufgabe übernehmen könnte. Auch vorhandene nationalstaatliche Organe, wie etwa die Rechnungshöfe oder die angesprochenen Normenkontrollräte, sind in dieser Frage fachlich nur begrenzt einsetzbar und im materiellen Zugriff wenig geeignet.

Die notwendigen Voraussetzungen richten sich in erster Linie auf eine erweiterte Sachkenntnis im Bereich der Aufgabenüberprüfung und der Regierungs- wie Verwaltungsorganisation, die sich ohne Einbezug des Wissenschaftsbereichs nur schwer realisieren lassen dürfte. Dies gilt insbesondere dann, wenn die Expertise sich nicht nur auf die europäische Ebene, sondern auch auf die einzelnen Mitgliedstaaten beziehen soll. Hinzu tritt die Erfordernis sachlicher und finanzieller Unabhängigkeit sowie ein dauerhaft gewährter Zugriff auf vermittelnde Einrichtungen und eine ungehinderte Berichterstattung. Dementsprechend wäre ein gesamteuropäisches Netzwerk bestehender und praxisnah arbeitender wissenschaftlicher Einrichtungen denkbar, das im Verbund die Europäische Union betrachtet und zugleich - unter Anwendung regelmäßiger peer-review-Verfahren - über die Entwicklungen in den jeweiligen Nationalstaaten berichtet. Die übergreifende Berichterstattung könnte sich an den wissenschaftlichen Routinen des Intergouvernementalen Ausschusses zum Klimawandel (IPCC) bei Vermeidung des hier gegebenen Totalitätsanspruches orientieren und durch regelmäßige Sachstandsberichte den Druck auf die Reformagenda aufrechterhalten.

Schließlich dürften sich die nationalen Parlamente, im Verbund mit dem Europäischen Parlament, am ehesten für die Rolle des Auftraggebers und der sachlich zuständigen Instanz eignen. Zum einen fällt diesen Einrichtungen traditionell die Rolle von Kontrollorganen gegenüber den jeweiligen Exekutiven zu, zum anderen verfügen sie über die notwendige Budgethoheit, die fachliche Expertise im Rahmen der Europa-Ausschüsse sowie über einen gewissen Anreiz, sich durch eigene Tätigkeit sowohl von den mitgliedstaatlichen Regierungen als auch von 
der europäischen Ebene abzugrenzen. Eine solche Funktion würde es den Parlamenten gestatten, verloren gegangenen Einfluss im Europäisierungsprozess zurückzugewinnen, einer sich schleichend verstärkenden Exekutivlastigkeit entgegenzuwirken, das europäische Demokratiedefizit zu lindern und schließlich das Potential der bereits im Primärrecht vorgesehenen Subsidiaritätsrüge über eine verbindende und unparteiische Instanz mit ernstzunehmendem Gehalt zu füllen.

\section{Ausblick}

In der Zusammenfassung zielen die hier vorgetragenen Empfehlungen vor allem auf ein „,besseres Europa“, das sich durch eine verstärkt funktionale Ausrichtung seiner Politiken auszeichnet, sich der gegebenen Bedarfe und positiven Routinen seiner Mitgliedstaaten versichert und auf der Anerkenntnis der eigenen Handlungs- und Tragfähigkeit basiert. Daraus folgt zunächst und unmittelbar weder ein „Mehr“ noch ein „Weniger“ an Europa, sondern primär die Erkenntnis, dass die europäische Integration nur dann zukunftsfähig sein kann, wenn sie sich auf jene Aufgaben konzentriert, die auf der EU-Ebene auch effektiv wahrgenommen werden können.

Die dafür vorzusehende Aufgabenüberprüfung würde den Integrationsprozess professioneller gestalten, unnötige Polarisierungen vermeiden und vor allem den „Völkern Europas“ eine erweiterte Transparenz und Mitwirkungsmöglichkeit erlauben, die weit über das hinausginge, was heute erkennbar ist. Eine entsprechende Anpassung des Aufgabenbestands (im Rahmen der aufgezeigten Vorgehensweisen und Umsetzungsmöglichkeiten) ist zwar nicht in kurzen Fristen zu verwirklichen, doch wird man sich der Diskussion nicht dauerhaft entziehen können. Auch dürfte nur eine konsequente und ggf. verstetigte Aufgabenkritik gewährleisten, dass populistische oder gar extremistische Positionen das Entwicklungspotential der Union nicht gefährden. Die Vertreter der Europäischen Union wie der Mitgliedstaaten wären im Ergebnis gut beraten, sich der in den vorliegenden Verträgen durchaus gegebenen Handlungsmöglichkeiten zu erinnern, um eine weitere Desillusionierung des Publikums, eine Einschränkung der eigenen Leistungsfähigkeit und vor allem eine Gefährdung des Erreichten zu vermeiden. 